\title{
BMJ Open How do primary healthcare workers in low-income and middle-income countries obtain information during consultations to aid safe prescribing? A systematic review protocol
}

Chris Smith, ${ }^{1,2}$ Michelle Helena van Velthoven, ${ }^{3}$ Neil Pakenham-Walsh ${ }^{4}$

To cite: Smith C, van Velthoven MH, PakenhamWalsh N. How do primary healthcare workers in lowincome and middle-income countries obtain information during consultations to aid safe prescribing? A systematic review protocol. BMJ Open 2019;9:e023015. doi:10.1136/ bmjopen-2018-023015

- Prepublication history and additional material for this paper are available online. To view these files, please visit the journal online (http://dx.doi. org/10.1136/bmjopen-2018023015).

Received 19 March 2018 Revised 15 October 2018 Accepted 30 November 2018

Check for updates

(c) Author(s) (or their employer(s)) 2019. Re-use permitted under CC BY-NC. No commercial re-use. See rights and permissions. Published by BMJ.

For numbered affiliations see end of article.

Correspondence to

Dr Chris Smith;

christopher.smith@Ishtm.ac.uk

\section{ABSTRACT}

Introduction There is a wide variety in prescribing by primary healthcare workers in low-income and middle-income countries. While there is much information available, both online and offline, there is variation in quality and relevance to different settings. Acting on incorrect or out-of-date information can lead to inappropriate prescribing and impact on patient safety. The aim of this review is to systematically review the evidence on how primary healthcare workers obtain information during consultations to prescribe safely and appropriately.

Methods and analysis We will identify relevant articles by searching electronic databases: Medline (Ovid), EMBASE (Ovid), Cochrane Central Register of Controlled Trials, CABI Global Health (Ovid), WHO global health library, POPLINE, Africa-Wide Information (Ebsco), Library, Information Science \& Technology Abstracts (Ebsco), ClinicalTrials.gov and WHO International Clinical Trials Registry Platform. Also, the Health Information For All network will be consulted and evidence databases (TRIP database, Epistemonikos, PDQ Evidence) will be searched. We will hand-search reference lists, run citation searches of included studies and email authors of identified papers. Observational and intervention studies involving primary healthcare workers in low-income and middle-income countries who prescribe and/or dispense medication will be included. The primary outcome is the proportion of healthcare workers obtaining information relevant to consultations from different sources. Secondary outcomes are the change in healthcare provider and patient knowledge or behaviour, adverse outcomes and use of resources. We will exclude studies focusing on secondary care. We anticipate a limited scope for meta-analysis and will provide a narrative overview of findings and tabular summaries of extracted data.

Ethics and dissemination No ethics approval is required. Findings will be disseminated through the Healthcare Information For All network.

PROSPERO registration number CRD42018091088.

\section{BACKGROUND}

Description of the issue

Accessible, high-quality primary healthcare is a key part of Universal Health Coverage, but is still not available almost 40 years after the Alma-Ata Declaration of $1978 .{ }^{12}$ Primary
Strengths and limitations of this study

- This review will systematically review the evidence on how primary healthcare workers obtain health information during consultations to support decision-making for prescribing in low-income and middle-income countries.

- Our review is following, where possible, the Cochrane Collaboration and Centre for Review and Dissemination methodology for conducting systematic reviews and we will report our findings based on guidelines from Preferred Reporting Items for Systematic Reviews and Meta-Analyses statement.

- A comprehensive search of the evidence will be conducted.

- A limitation of the review is that the quality and quantity of studies with similar interventions conducted in low-income and middle-income countries may be limited. Therefore, it is unlikely that we will be able to conduct a meta-analysis.

- The review results will be disseminated to inform efforts that aim to improve the availability of health information in low-income and middle-income countries.

healthcare workers in low-income and middle-income settings are often faced with large numbers of consultations in remote rural areas. Also, in addition to infectious diseases, such as HIV, TB and malaria, there is an increasing prevalence of non-communicable diseases and mental health issues. ${ }^{3}$ This forces health workers to deal with more complex treatment of patients, often with limited support and resources, on a background of having received varied levels of education and training. ${ }^{4-6}$

Primary healthcare workers encompass a diverse range of healthcare cadres such as doctors, clinical officers, nurses, pharmacists and drug store vendors and are often the first level of contact of individuals, the family and 
the community with the national health system. ${ }^{2}$ Poor performance of these primary healthcare workers can result in incorrect diagnosis and management of common diseases, including inappropriate drug prescribing.

There are various issues related to inappropriate and unsafe prescribing. There are medication errors which applies to errors of dose or route of administration, and to errors of communication between prescription and dispensing (eg, bad handwriting, etc). Also, healthcare workers can prescribe the wrong medicine, with or without a failure to correctly diagnose the case. Furthermore, overprescribing is a huge problem, especially in low-income and middle-income countries (LMICs), with almost every patient being given one or more medicines per consultation. ${ }^{7}$ Overuse of antimicrobials accelerates the emergence and growth of antimicrobial resistance which is an increasingly serious threat to global public health. 'Rational' use of medicines depends largely on the ability of the health worker to make the correct diagnosis and then prescribe (or not) accordingly. ${ }^{9}$

Unsafe prescribing by primary healthcare workers have often been attributed to weak medication systems, poor environmental conditions or staff shortages, poor education, inadequate training and lack of knowledge and skills. ${ }^{10}$ Previous studies in Africa have suggested variations in knowledge about the basics on how to diagnose and manage common diseases, going right across the health workforce and often associated with suboptimal, ineffective and dangerous healthcare practices. ${ }^{911-13}$

One important factor influencing knowledge and practice of primary healthcare workers is the wide variation in quality of health information and relevance to different settings with respect to their language, geographical focus and technical level. Offline resources may be out-of-date and might not have not incorporated changes in medical knowledge and guidance that have occurred over time such as new diagnostic tests and antibiotic regimens. Acting on such information can lead to incorrect diagnosis and inappropriate prescribing.

\section{Description of the intervention}

By 'healthcare information' we refer to the information that healthcare workers need at the point of care to guide them in prescribing: to guide them on how to select whether to prescribe a medicine for an individual patient, which medicine to use and how to do so.

For this study, we are focusing on healthcare information resources such as point-of-care decision tools, formularies, books, manuals, guidelines and protocols, rather than routine health information such as patient records and local epidemiological reports.

\section{How the intervention might work}

Arguably, correct information to guide prescribing (including diagnosis and, where appropriate, selection of medicine) is the single most important aspect of point-ofcare information for most of clinical consultations, especially at primary care level.
Factors influencing whether healthcare providers attempt to access information during consultations include previous training and availability of the information which could include internet access. ${ }^{911} 14$ Additional influencing factors might be how common or rare a condition is, whether it is a condition where it is important to give correct medication/dose (ie, risk or adverse event or child dosing required), the degree of risk health workers are prepared to take without checking and 'black holes', that is, not aware of lack of knowledge.

\section{Why is it important to do this review}

The WHO aims to reduce the level of severe, avoidable harm related to medications by $50 \%$ over the next 5 years, globally. ${ }^{10}$ While there exists a plethora of health information, it is unclear to what extent primary healthcare workers in LMICs access such information to aid prescribing, including free and for purchase materials, both in digital and offline forms. A recent systematic review found that most of the interventions to improve healthcare worker performance have focused on 'supervision' as opposed to 'tools and aids' such as protocols and/or charts. Digital health tools targeting primary healthcare workers were not reported to have been extensively studied. ${ }^{6}$

To our knowledge, no systematic reviews have assessed how primary healthcare workers in LMICs obtain health information during consultations to aid safe prescribing. Therefore, the aim of this review is to review the evidence on how primary healthcare workers obtain health information during consultations to support prescribing decision-making.

\section{Objectives}

The objective is to systematically review the evidence on how primary healthcare workers obtain health information during consultations to aid decision-making for prescribing in LMICs and its impact by assessing:

1. Proportions of healthcare workers obtaining healthcare information from different sources (eg, use of books, guidelines, digital resources, peer networks) during consultations (ie, from the time when the patient arrives to leaving) to make decisions on prescribing.

2. Change in healthcare provider and patient knowledge or behaviour.

3. Adverse outcomes (eg, misconceptions resulting from out-of-date or incorrect information, whether obtaining information to guide prescribing relevant to a consultation reduces the quality of a consultation, for example patients trust a healthcare worker less or perceive a lack of empathy when a health worker looks up information).

4. Use of resources.

\section{METHODS}

This is the protocol for a systematic review of the literature that is reported, where possible, according to the Preferred Reporting Items for Systematic Reviews and 
Meta-Analyses for Protocols as provided in online supplementary file $1 .{ }^{15}$ Our review is following, where possible, the Cochrane Collaboration ${ }^{16}$ and Centre for Review and Dissemination ${ }^{17}$ methodology for conducting systematic reviews where possible.

\section{Criteria for considering studies \\ Types of studies}

We will include observational studies, such as cross-sectional surveys, cohort studies, qualitative studies (eg, interview studies and focus groups) and mystery client studies, and intervention studies, such as randomised controlled trials (RCTs) and non-randomised studies (non-RCTs, controlled before-and-after studies, interrupted-time-series studies). We will only include studies reported in English that are published after 2000.

\section{Types of participants}

We will include studies involving primary healthcare workers in LMICs who prescribe or dispense medication, or order medical tests, such as doctors, clinical officers, nurses, midwifes, pharmacists, specialist drug shop vendors or community health workers. We will exclude studies focusing on secondary care or hospital settings, allied health professionals, for example, physiotherapists and traditional medicine health workers.

\section{Types of interventions}

We will include any studies assessing interventions to improve access to healthcare information during consultation for prescribing, for example, tools and aids (possible interventions could use digital resources, books, protocols, etc). Also, studies that do not focus on an intervention (eg, observational studies) will be included. We will not include quality improvement, supervision or mentoring interventions unless support in the form of tools or aids for healthcare workers are explicitly available during the consultation.

\section{Types of comparators}

We will include any type of comparator interventions.

\section{Types of outcome measures}

The primary outcome of this review is the proportion of healthcare workers obtaining healthcare information during consultations (ie, from the time when the patient arrives to leaving) from different sources (eg, use of books, guidelines, digital resources, peer networks, no information-memory). Secondary outcomes are the change in healthcare provider knowledge or behaviour (eg, prescribing/diagnosis, intervention studies), change in patient knowledge, behaviour or clinical outcomes, adverse outcomes and use of particular resources.

\section{Information sources}

Relevant articles will be identified by searching electronic databases:

- MEDLINE through Ovid.

- EMBASE through Ovid.
- CABI Global health through Ovid.

- WHO global health library (http://www.globalhealthlibrary.net/php/index.php).

- POPLINE(http://www.popline.org/).

- Africa-Wide Information (https://www. ebsco.com/ products / research-databases / africa-wide-information).

- Library, Information Science \& Technology D/base (https://www.ebsco.com/products/research-databases/library-information-science-and-technologyabstracts).

- Web of Science.

- Cochrane Central Register of Controlled Trials (CENTRAL) (http://www.cochranelibrary.com/ about/central-landing-page.html).

- WHO International Clinical Trials Registry Platform (http://apps.who.int/trialsearch/).

\section{- ClinicalTrials.gov}

In addition, the Healthcare Information For All network will be consulted for relevant publications and evidence databases such as TRIP database (https:// www.tripdatabase.com/), Epistemonikos (https://www. epistemonikos.org/) and PDQ Evidence (https://www. pdq-evidence.org/). We will hand-search reference lists, run citation searches of included studies (eg, in Google Scholar, Scopus or Web of Knowledge) and email authors of identified papers to identify additional articles.

\section{Search strategy}

Final search strategies can be found in online supplementary file 2 tailored to the different databases with a medical research librarian (John Eyers). No study design filter will be used as both quantitative and qualitative studies are included. We will use the titles, abstracts and keywords of a set of articles that we know that meet our inclusion criteria to define a search strategy that will return all these articles without an unmanageably large number of irrelevant articles.

\section{Data management, collection and analysis \\ Selection of studies}

We will exclude duplicate references by comparing titles, authors and digital object identifiers between similar search results using two software programs (Endnote: http://endnote.com/ and EPPI: http://eppi.ioe.ac.uk/ $\mathrm{cms} /$ ). One reviewer will screen titles/abstracts of search results against the inclusion and exclusion criteria and $10 \%$ of the titles/abstracts will be screened by a second reviewer for validation. One reviewer will retrieve a fulltext paper when a study includes participants who are primary healthcare workers in LMICs and it assesses one or more relevant outcome measures. Two reviewers will assess full text for eligibility, with any disagreement to be resolved through discussion with a third author.

\section{Data extraction}

To extract data from included studies, we will use a standardised Excel form that includes general information 
(title, authors, date, etc), study characteristics (study design, aim, duration, inclusion/exclusion criteria), risk of bias (depending on study design), participants (description, geographical location setting, etc), intervention (if appropriate and to include sources of online or offline information sources used), outcomes (as specified above, other outcomes, adverse events) and results (outcomes, times of assessment). We will pilot the data extraction form on a small number of studies to develop the final data extraction form. One reviewer will extract data from included studies that will be validated by a second reviewer.

\section{Assessment of risk of bias}

Risk of bias for RCTs will be undertaken by one reviewer and independently checked by another. Any disagreements will be resolved by consensus and the opinion of a third reviewer. The methods specified in the Cochrane Collaboration tool for assessing risk of bias will be used which includes assessment of the following six domains: random sequence generation, allocation concealment, blinding of participants and personnel, blinding of outcome assessment, incomplete outcome data and selective reporting. Three bias assessment categories will be used: low, high and unclear risk. As specified in the Cochrane handbook, ${ }^{16}$ an adapted version of these domains will also be used for non-randomised studies (NRS). For case-control and cohort studies, the Newcastle-Ottawa Scales will be considered. For observational studies, guidance from the Centre for Review and Dissemination ${ }^{17}$ will be used to inform the quality assessment. At the moment, the value of quality assessment of qualitative research is debated. A relevant tool will be selected based on the Cochrane Handbook's recommendations.

\section{Assessment of heterogeneity}

We anticipate a limited scope for meta-analysis due to differences in study populations, interventions and outcomes. If a sufficient number of studies are found, we will explore heterogeneity through consideration of the study populations, methods and interventions, by visual inspection of results. Also in statistical terms, we will assess the $\chi^{2}$ test for homogeneity and the $\mathrm{I}^{2}$ statistic. We define statistically significant heterogeneity as $\mathrm{p}<0.10$. The $\mathrm{I}^{2}$ will be assessed with the following levels of inconsistency: $\mathrm{I}^{2}$ of $0 \%-25 \%=$ lowlevel of inconsistency, $26 \%-50 \%=$ moderate level of inconsistency and $>50 \%=$ high level of inconsistency.

\section{Data synthesis}

We will provide a narrative overview of findings and tabular summaries of extracted data. If a meta-analysis can be performed, this will estimate a summary measure of effect on relevant outcomes. For dichotomous outcomes, ORs will be used as the summary statistic. For continuous outcomes, mean difference will be the summary statistic. Meta-analyses will be conducted when clinically homogeneous studies of similar comparisons reporting the same outcome measures are identified. Standard pairwise meta-analysis will be conducted when more than one RCT is identified. Quality of evidence will be assessed using the Grading of Recommendations, Assessment, Development and Evaluations approach.

\section{Subgroup analyses}

If appropriate, we will provide a narrative overview of subgroups including different interventions (eg, digital vs analogue), cadres of healthcare workers and geographical regions.

\section{Ethics and dissemination}

We will submit the full systematic review for publication in a peer-reviewed medical journal. The review will inform efforts that aim to improve the prescribing practices of healthcare workers in LMICs. Findings will be disseminated through the Health Information For All network.

\section{Patient and public involvement}

Patients and the public were not involved in writing this protocol.

\section{Author affiliations}

${ }^{1}$ Graduate School of Tropical Medicine \& Global Health (TMGH), Nagasaki University, Nagasaki, Japan

${ }^{2}$ Department of Clinical Research, London School of Hygiene \& Tropical Medicine, London, UK

${ }^{3}$ Healthcare Translation Research Group, Department of Paediatrics, University of Oxford, Oxford, UK

${ }^{4}$ Healthcare Information For All (HIFA) network, Oxford, UK

Acknowledgements We are grateful to Tom Burnham, Sarah Cavanagh, Rita Reyburn and Lorenzo Moja for their comments on the manuscript, and to John Eyers for advising on and conducting the database searches.

Contributors CS and MHvV jointly wrote the protocol. NP-W provided extensive comments on the protocol.

Funding This work was supported by Nagasaki University.

Disclaimer The funder did not have input in developing this protocol.

Competing interests MHvV is the director of Dutches Consulting Ltd which provides advice to clients in the life sciences.

Patient consent for publication Not required.

Ethics approval No ethics approval is required.

Provenance and peer review Not commissioned; externally peer reviewed.

Open access This is an open access article distributed in accordance with the Creative Commons Attribution Non Commercial (CC BY-NC 4.0) license, which permits others to distribute, remix, adapt, build upon this work non-commercially, and license their derivative works on different terms, provided the original work is properly cited, appropriate credit is given, any changes made indicated, and the use is non-commercial. See: http://creativecommons.org/licenses/by-nc/4.0/.

\section{REFERENCES}

1. Kruk ME, Larson E, Twum-Danso NA. Time for a quality revolution in global health. Lancet Glob Health 2016;4:e594-e596.

2. WHO. Declaration of alma-atainternational conference on primary health care, Alma-Ata, USSR, 6-12September 1978. 1978 http:// www.who.int/publications/almaata_declaration_en.pdf.

3. Economist. The next pandemic: non-communicable diseases in developing countries. $2017 \mathrm{http}: / /$ accessaccelerated.economist. com/reports/thenextpandemic/.

4. Wafula FN, Miriti EM, Goodman CA. Examining characteristics, knowledge and regulatory practices of specialized drug shops in 
Sub-Saharan Africa: a systematic review of the literature. BMC Health Serv Res 2012;12:223.

5. Tumwikirize WA, Ekwaru PJ, Mohammed K, et al. Management of acute respiratory infections in drug shops and private pharmacies in Uganda: a study of counter attendants' knowledge and reported behaviour. East Afr Med J 2004;:S33-40.

6. Vasan A, Mabey DC, Chaudhri S, et al. Support and performance improvement for primary health care workers in low- and middleincome countries: a scoping review of intervention design and methods. Health Policy Plan 2017;32:437-52.

7. Krause G, Borchert M, Benzler J, et al. Rationality of drug prescriptions in rural health centres in Burkina Faso. Health Policy Plan 1999;14:291-8.

8. WHO. Antimicrobial resistance. http://www.who.int/mediacentre/ factsheets/fs194/en/.

9. Pakenham-Walsh N, Bukachi F. Information needs of health care workers in developing countries: a literature review with a focus on Africa. Hum Resour Health 2009;7:30.
10. WHO. WHO global patient safety challenge: medication without harm. 2017http://www.who.int/patientsafety/medication-safety/ medication-without-harm-brochure/en/.

11. Pakenham-Walsh N, Priestly C, Smith R. Meeting the information needs of health workers in developing countries. BMJ 1997;314:90.

12. Macrorie R. Empowering doctors in the developing world. BMJ 1997;314:980.

13. Sekikawa A, Laporte RE, Satoh T, et al. Health workers need information from countries with better health indicators than Britain and the US. BMJ 1997;314:1418.

14. Jafar TH, Jessani S, Jafary FH, et al. General practitioners' approach to hypertension in urban Pakistan: disturbing trends in practice. Circulation 2005;111:1278-83.

15. Shamseer L, Moher D, Clarke M, et al. Preferred reporting items for systematic review and meta-analysis protocols (PRISMA-P) 2015: elaboration and explanation. BMJ 2015;349:g7647.

16. Cochrane Training. Cochrane Handbook for Systematic Reviews of Interventions, 2011. https://training.cochrane.org/handbook.

17. CRD. Systematic Reviews CRD's guidance for undertaking reviews in health care. 2010 www.york.ac.uk/inst/crd/index_guidance.htm. 